\title{
SEARCH FOR COSMIC NEUTRINO POINT SOURCES WITH FOUR YEARS OF DATA FROM THE ANTARES TELESCOPE
}

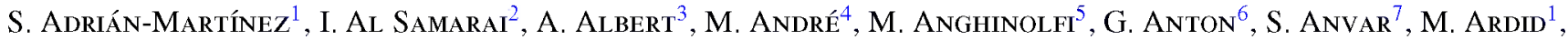
T. Astraatmadja ${ }^{8.39}$, J-J. AuberT ${ }^{2}$, B. BareT ${ }^{9}$, S. Basa ${ }^{11}$, V. Bertin ${ }^{2}$, S. Biagi ${ }^{11,12}$, C. Bigongiari ${ }^{13}$, C. Bogazzi $^{8}$,

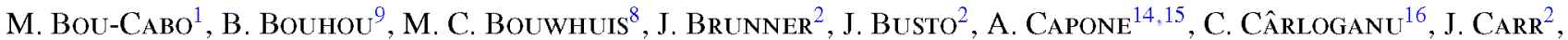

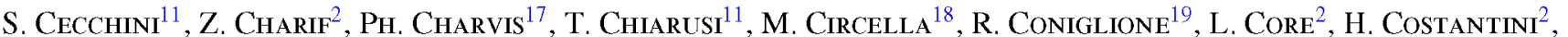
P. Coyle $^{2}$, A. Creusot ${ }^{9}$, C. Curtil ${ }^{2}$, G. De Bonis ${ }^{14,15}$, M. P. DeCowski ${ }^{8}$, I. Dekereser ${ }^{21}$, A. Deschamps ${ }^{17}$, C. Distefano ${ }^{19}$, C. Donzaud ${ }^{9.21}$, D. Dornic ${ }^{2,13}$, Q. Dorosti ${ }^{22}$, D. Drouhin ${ }^{3}$, T. Eberi ${ }^{6}$, U. EMANuele $^{13}$, A. EnZenhö̈er $^{6}$, J-P. ERnenWein $^{2}$, S. Escoffier ${ }^{2}$, K. Fehn ${ }^{6}$, P. Fermani ${ }^{14,15}$, M. Ferri ${ }^{1}$, S. Ferri ${ }^{23}$, V. Flaminio ${ }^{24.25}$, F. Folger ${ }^{6}$, U. Fritschi ${ }^{6}$, J-L. Fuda ${ }^{21}$, S. Galatì ${ }^{2}$, P. Gay ${ }^{16}$, K. Geyer ${ }^{6}$, G. Giacomelli ${ }^{11,12}$, V. Giordano ${ }^{19}$, A. Gleixner ${ }^{6}$, J. P. Gómez-González ${ }^{13}$, K. Graf ${ }^{6}$, G. Guillard ${ }^{16}$, G. Hallewell ${ }^{2}$, M. Hamal ${ }^{26}$, H. VAN Haren ${ }^{27}$, A. J. HeiJboer ${ }^{8}$, Y. Hello ${ }^{17}$, J. J. Hernández-ReY ${ }^{13}$,

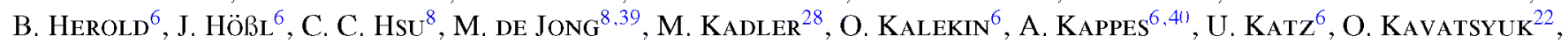

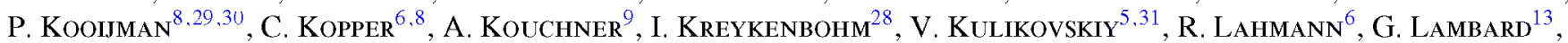

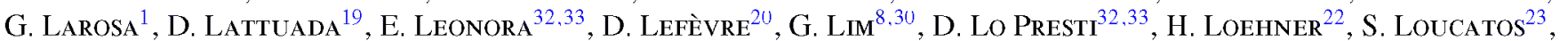
F. Louis ${ }^{7}$, S. Mangano ${ }^{13}$, M. Marcelin ${ }^{10}$, A. Margiotta ${ }^{11,12}$, J. A. Martínez-Mora ${ }^{1}$, A. Meli $^{6}$, T. Montaruli $^{18,34}$, M. Morganti ${ }^{24,41}$, H. Motz ${ }^{6}$, M. NefF ${ }^{6}$, E. Nezri ${ }^{10}$, D. Palioselitis ${ }^{8}$, G. E. PăVĂLaş ${ }^{35}$, K. Payet ${ }^{23}$, J. Petrovic ${ }^{8}$,

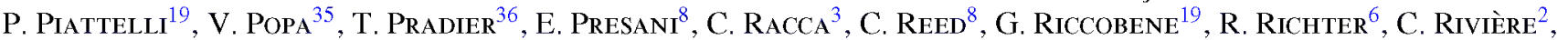

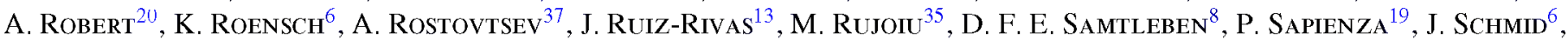

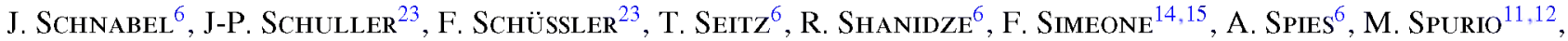

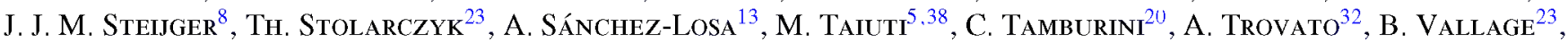

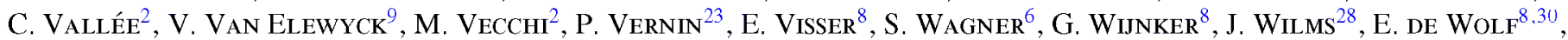
H. YEPES ${ }^{13}$, D. ZABOROV ${ }^{37}$, J. D. ZORNOZA ${ }^{13}$, AND J. ZÚÑIGA ${ }^{13}$

${ }^{1}$ Institut d'Investigació per a la Gestió Integrada de les Zones Costaneres (IGIC) - Universitat Politècnica de València. C/Paranimf 1, E-46730 Gandia, Spain ${ }^{2}$ CPPM, Aix-Marseille Université, CNRS/IN2P3, Marseille, France

${ }^{3}$ GRPHE - Institut universitaire de technologie de Colmar, 34 rue du Grillenbreit, BP 50568 - 68008 Colmar, France

4 Technical University of Catalonia, Laboratory of Applied Bioacoustics, Rambla Exposició, E-08800 Vilanova i la Geltrú, Barcelona, Spain ${ }^{5}$ INFN - Sezione di Genova, Via Dodecaneso 33, I-16146 Genova, Italy

${ }^{6}$ Friedrich-Alexander-Universität Erlangen-Nürnberg, Erlangen Centre for Astroparticle Physics, Erwin-Rommel-Str. 1, D-91058 Erlangen, Germany

${ }^{7}$ Direction des Sciences de la Matière - Institut de recherche sur les lois fondamentales de l'Univers - Service d'Electronique des Détecteurs et d'Informatique, CEA Saclay, F-91191 Gif-sur-Yvette Cedex, France

${ }^{8}$ Nikhef, Science Park, Amsterdam, The Netherlands

${ }^{9}$ APC - Laboratoire AstroParticule et Cosmologie, UMR 7164 (CNRS, Université Paris 7 Diderot, CEA, Observatoire de Paris) 10 , rue Alice Domon et Léonie Duquet, F-75205 Paris Cedex 13, France

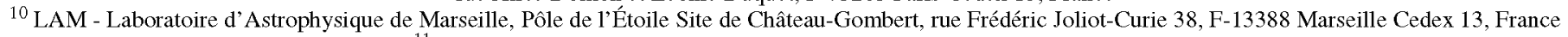
${ }^{11}$ INFN - Sezione di Bologna, Viale Berti-Pichat 6/2, I-40127 Bologna, Italy

12 Dipartimento di Fisica dell'Università, Viale Berti Pichat 6/2, I-40127 Bologna, Italy

${ }^{13}$ IFIC - Instituto de Física Corpuscular, Edificios Investigación de Paterna, CSIC - Universitat de València, Apdo. de Correos 22085 , E-46071 Valencia, Spain 14 INFN -Sezione di Roma, P.le Aldo Moro 2, I-00185 Roma, Italy

15 Dipartimento di Fisica dell'Università La Sapienza, P.le Aldo Moro 2, I-00185 Roma, Italy

${ }^{16}$ Clermont Université, Université Blaise Pascal, CNRS/IN2P3, Laboratoire de Physique Corpusculaire, BP 10448 , F-63000 Clermont-Ferrand, France

${ }^{17}$ Géoazur - Université de Nice Sophia-Antipolis, CNRS/INSU, IRD, Observatoire de la Côte d'Azur and Université Pierre et Marie Curie, BP 48, F-06235 Villefranche-sur-mer, France

${ }^{18}$ INFN - Sezione di Bari, Via E. Orabona 4, I-70126 Bari, Italy

${ }^{19}$ INFN - Laboratori Nazionali del Sud (LNS), Via S. Sofia 62, I-95123 Catania, Italy

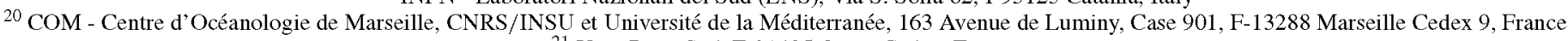
${ }^{21}$ Univ Paris-Sud, F-91405 Orsay Cedex, France

${ }^{22}$ Kernfysisch Versneller Instituut (KVI), University of Groningen, Zernikelaan 25, 9747 AA Groningen, The Netherlands

${ }^{23}$ Direction des Sciences de la Matière - Institut de recherche sur les lois fondamentales de l'Univers - Service de Physique des Particules, CEA Saclay, F-91191 Gif-sur-Yvette Cedex, France

24 INFN - Sezione di Pisa, Largo B. Pontecorvo 3, I-56127 Pisa, Italy

25 Dipartimento di Fisica dell'Università, Largo B. Pontecorvo 3, I-56127 Pisa, Italy

${ }^{26}$ University Mohammed I, Laboratory of Physics of Matter and Radiations, BP 717, Oujda 6000, Morocco

${ }^{27}$ Royal Netherlands Institute for Sea Research (NIOZ), Landsdiep 4,1797 SZ 't Horntje (Texel), The Netherlands

${ }^{28}$ Dr. Remeis-Sternwarte and ECAP, Universität Erlangen-Nünberg, Sternwartstr. 7, D-96049 Bamberg, Germany

${ }^{29}$ Universiteit Utrecht, Faculteit Betawetenschappen, Princetonplein 5, 3584 CC Utrecht, The Netherlands

${ }^{30}$ Universiteit van Amsterdam, Instituut voor Hoge-Energie Fysica, Science Park 105, 1098 XG Amsterdam, The Netherlands

31 Moscow State University, Skobeltsyn Institute of Nuclear Physics, Leninskie gory, 119991 Moscow, Russia

32 INFN - Sezione di Catania, Viale Andrea Doria 6, I-95125 Catania, Italy

${ }^{33}$ Dipartimento di Fisica ed Astronomia dell'Università, Viale Andrea Doria 6, I-95125 Catania, Italy

34 Département de Physique Nucléaire et Corpusculaire, Université de Genève, 1211, Geneva, Switzerland

35 Institute for Space Sciences, R-77125 Bucharest, Măgurele, Romania

${ }^{36}$ IPHC-Institut Pluridisciplinaire Hubert Curien - Université de Strasbourg et CNRS/IN2P3 23 rue du Loess, BP 28 , F-67037 Strasbourg Cedex 2, France

${ }^{37}$ ITEP - Institute for Theoretical and Experimental Physics, B. Cheremushkinskaya 25, 117218 Moscow, Russia

${ }^{38}$ Dipartimento di Fisica dell'Università, Via Dodecaneso 33, I-16146 Genova, Italy

Received 2012 July 12; accepted 2012 October 3; published 2012 November 2 


\begin{abstract}
In this paper, a time-integrated search for point sources of cosmic neutrinos is presented using the data collected from 2007 to 2010 by the ANTARES neutrino telescope. No statistically significant signal has been found and upper limits on the neutrino flux have been obtained. Assuming an $E_{v}^{-2}$ spectrum, these flux limits are at $1-10 \times 10^{-8} \mathrm{GeV} \mathrm{cm}^{-2} \mathrm{~s}^{-1}$ for declinations ranging from $-90^{\circ}$ to $40^{\circ}$. Limits for specific models of RX J1713.7-3946 and Vela X, which include information on the source morphology and spectrum, are also given.
\end{abstract}

Key words: astroparticle physics - cosmic rays - neutrinos

Online-only material: color figures

\section{INTRODUCTION}

One of the main goals of the ANTARES telescope (Ageron et al. 2011) is the detection of cosmic neutrinos and the identification of their sources. Neutrinos only interact via the weak interaction and are stable, making them unique probes to study the high-energy universe. The production of high-energy neutrinos has been proposed (Halzen \& Hooper 2002; Bednarek et al. 2005; Stecker 2005) for several kinds of astrophysical sources in which the acceleration of hadrons may occur. In the interaction of cosmic rays (CRs) with matter or radiation, charged pions are produced. The decay of these charged pions yields neutrinos and muons. The subsequent decay of the latter produces in turn more neutrinos. While CRs, except at ultra high energies, are deflected by galactic and intergalactic magnetic fields, neutrinos point back to their sources. Therefore, the detection of neutrinos may give valuable information on the origin of CRs. It would also settle the question of the hadronic versus leptonic mechanism in several sources from which highenergy gamma rays have been observed (Berezhko et al. 2008).

The best neutrino flux upper limits up to PeV energies for the Southern Hemisphere have been established by the ANTARES experiment using 2007-2008 data (Adrián-Martínez et al. 2011). In the present paper, this analysis is extended by adding two more years of data with the full configuration of 12 detection lines. Furthermore, the information on the amount of light produced in the events, which is a quantity correlated to the neutrino energy and which helps to distinguish the atmospheric neutrino background from a potential high-energy signal, is taken into account.

The structure of this paper is as follows. In Section 2 the ANTARES detector is briefly described. Sections 3 and 4 describe the online selection and the simulation, respectively. The track reconstruction is explained in Section 5. The selection of events is described in Section 6. Section 7 is devoted to the evaluation of the detector performance. The search method and the limit setting are described in Sections 8 and 9. Section 10 shows how the search is improved by including the energy information. Results are presented in Section 11. A cross-check based on an alternative method is explained in Section 12. Finally, the conclusions are summarized in Section 13.

\section{THE ANTARES DETECTOR}

The operation principle of neutrino telescopes is based on the detection of the Cherenkov light induced by relativistic muons produced in the charged current (CC) weak interactions of highenergy neutrinos close to or inside the detector. The information

\footnotetext{
${ }^{39}$ Also at University of Leiden, the Netherlands.

${ }^{40}$ On leave of absence at the Humboldt-Universität zu Berlin.

41 Also at Accademia Navale de Livorno. Livorno. Italy.
}

on the time and position of the detected photons is used to reconstruct the muon trajectory, which is correlated with the direction of the incoming neutrino. Other signatures are also possible, such as the cascades produced in the CC interactions of electron and tau neutrinos and in the neutral current interactions of all neutrino flavors. In this analysis muons induced by high-energy neutrinos are used. For these events, the detector acceptance is large due to the long muon range and the neutrino direction is derived with an accuracy of a fraction of a degree.

The construction of the ANTARES detector (Ageron et al. 2011) was completed in 2008, after several years of site exploration and detector R\&D (Amram et al. 2002; Aguilar et al. 2005a, 2011a). The detector is located at $\left(42^{\circ} 48^{\prime} \mathrm{N}, 6^{\circ} 10^{\prime}\right.$ E) at a depth of $2475 \mathrm{~m}$, in the Mediterranean Sea, $40 \mathrm{~km}$ from the French town of Toulon. It comprises a three-dimensional array of 885 optical modules (OMs) looking $45^{\circ}$ downward and distributed along 12 vertical detection lines. An OM (Amram et al. 2002) consists of a 10 inch photomultiplier (PMT) housed in a glass sphere together with its base, a special gel for optical coupling and a $\mu$-metal cage for magnetic shielding. The OMs are grouped in 25 triplets (or storeys) on each line, except for one of the lines on which acoustic devices are installed (Aguilar et al. 2011b) and which therefore contains only 20 optical storeys. The total length of each line is $450 \mathrm{~m}$; these are kept taut by a buoy located at the top of the line. The lower $100 \mathrm{~m}$ are not instrumented. The distance between triplets is $14.5 \mathrm{~m}$ and the separation between the lines ranges from 60 to $75 \mathrm{~m}$. The lines are connected to a central junction box, which in turn is connected to shore via an electro-optical cable. Figure 1 shows a schematic view of the detector.

The detector also includes several calibration systems. The lines slowly move due to the sea current (up to $\sim 15 \mathrm{~m}$ at the top of the line in case of currents of $20 \mathrm{~cm} \mathrm{~s}^{-1}$ ). A set of acoustic devices together with tiltmeters and compasses along the lines are used to reconstruct the shape of the lines and orientation of the storeys every two minutes (Adrián-Martínez et al. 2012). The acoustic system provides the position of each optical module with a precision better than $15 \mathrm{~cm}$. The time calibration is performed by means of a master clock on shore and a set of optical beacons (four along each line). This allows for a calibration of the time offsets of the photomultipliers with a precision better than $1 \mathrm{~ns}$ (Aguilar et al. 2011a).

In this analysis, data from 2007 January 29 to 2010 November 14 are used. The total integrated live-time is 813 days, out of which 183 correspond to the five-line period. Causes of loss of efficiency are periods of high bioluminescence and sea operations.

\section{ONLINE SELECTION}

The charge and time information of all signals from the PMTs which exceed a pre-defined threshold voltage, typically the 


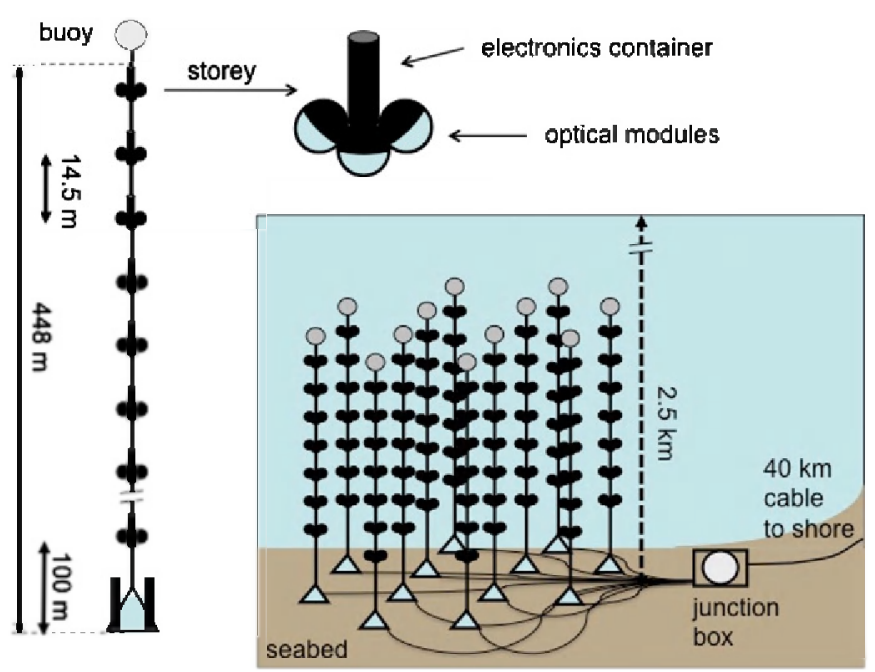

Figure 1. Schematic view of the ANTARES detector, consisting of 12 mooring lines connected to the shore station through an electro-optical cable. (A color version of this figure is available in the online journal.)

equivalent to 0.3 single photoelectrons (Aguilar et al. 2007), are first digitized into "hits" and then sent to shore where they are filtered by a farm of PCs. For this analysis, two different filter algorithms were used to select the events. Both are based on the assumption that the optical background processes such as potassium-40 radioactive decays and bioluminescence are not correlated and induce single photoelectron hits. Hence, a first selection of the signal requires hits with a high charge (usually $>3$ photoelectrons) or coincident hits within a time window of $20 \mathrm{~ns}$ on separate OMs of the same storey (L1 hits). The first trigger requires at least five L1 hits compatible with a muon track in any direction. The second trigger is defined as the occurrence of at least two L1 hits in three consecutive storeys within a specific time window. This time window is $100 \mathrm{~ns}$ in the case where the two storeys are adjacent and 200 ns for next-to-adjacent storeys. In addition to the events selected by the trigger, the singles count rate of each OM is stored.

\section{SIMULATIONS}

Simulations are required for determining the acceptance and angular resolution of the detector, since in the absence of a source these quantities cannot readily be measured.

The event simulation starts with the generation of upgoing muon neutrino events using the GENHEN package (Bailey 2002), which uses CTEQ6D (Pumplin et al. 2002) parton density functions for computing the deep inelastic charged current neutrino scattering cross section. The events are weighted according to the cross section and their probability to survive the passage through the Earth. If the neutrino interaction occurs near the detector, the hadronic shower resulting from the break-up of the target nucleon is simulated using GEANT (Brunner 2003). Otherwise, only the resulting muon is propagated to the detector using the MUSIC code (Antonioli et al. 1997). Atmospheric muons reconstructed as upgoing are a source of background for a neutrino signal and their rejection is a crucial point in this analysis as will be described in Section 6. Downgoing atmospheric muons were simulated with the program MUPAGE (Carminati et al. 2008; Becherini et al. 2006) which provides parameterized muons and muon bundles at the detector.

Inside the detector, the Cherenkov photons emitted along a muon track and arriving on the OMs are simulated by sampling tabulated values of photon arrival times. The arrival time distributions have been derived by tracking individual photons taking into account the measured absorption and scattering parameters (Aguilar et al. 2005b).

The PMT transit time spread (TTS) is simulated by a Gaussian smearing of photon arrival times. Optical backgrounds are added to the events according to the measured rates observed in the count rate data. Similarly, simulated hits from inactive OMs are deleted from the event. Sampling the count rate data from the runs selected for the analysis ensures that the simulation contains the same background and detector conditions as the analyzed data set. The same trigger algorithms are applied to the simulation and the data.

An uncertainty of $50 \%$ on the atmospheric muon flux is estimated using the same procedure described in Aguilar et al. (2010). For the atmospheric neutrino flux, a systematic uncertainty of $30 \%$ is considered (Barr et al. 2006).

\section{RECONSTRUCTION}

Tracks are reconstructed from the hits in the triggered events using a multi-step algorithm (see Heijboer 2004 for a more detailed description). The initial steps provide a starting point for the final maximization of the track likelihood. The likelihood is defined as the probability density of the observed hit time residuals, $r$, given the track parameters (position at some arbitrary time and direction). The time residual $r$ is defined as the difference between the observed and expected hit time for the assumed track parameters.

It was found that the likelihood function has many local maxima and that the maximization procedure needs to be started with track parameters close to the optimal values. The initial steps in the algorithm provide this near-optimal solution, estimating the track parameters using increasingly refined score functions: a linear $\chi^{2}$ fit, a so-called M-estimator minimizing $g(r)=\sqrt{1+r^{2}}$ and a simplified version of the full likelihood fit. Each fit uses increasingly more inclusive hit selections based on the preceding stage. This sequence is started at nine different starting points to further increase the probability of finding the global optimum. Using more than nine starting points was found to have only marginal impact on the reconstruction quality and was not deemed worth the additional processing time.

The final likelihood function uses parameterizations for the probability density function (pdf) of the signal hit time residual, derived from simulations. The pdfs also include hits arriving late due to Cherenkov emission by secondary particles or light scattering. Furthermore, the probability of a hit being due to background is accounted for.

The quality of the track fit is quantified by the parameter

$$
\Lambda \equiv \frac{\log (L)}{N_{\text {hits }}-5}+0.1 \times\left(N_{\text {comp }}-1\right)
$$

which incorporates the maximum value of the likelihood, $L$, and the number of degrees of freedom of the fit, i.e., the number of hits, $N_{\text {hits }}$, used in the fit minus the number of fit parameters; $N_{\text {comp }}$ is the number of times the repeated initial steps of the reconstruction converged to the same result. In general, $N_{\text {comp }}=1$ for badly reconstructed events while it can be as large as nine for well reconstructed events. The coefficient 0.1 in Equation (1) was chosen to maximize the separation in $\Lambda$ between simulated signal and misreconstructed downgoing muons.

The $\Lambda$ variable can be used to reject badly reconstructed events, in particular atmospheric muons that are 
Table 1

Number of Events before and after Applying Selection Cuts for Data (Second Column) and Monte Carlo Simulations (Third. Fourth. and Fifth Columns)

\begin{tabular}{lccc}
\hline \hline & Data & Atm. $\mu$ & $E_{v}^{-2} v$ \\
\hline Triggered events & $3.94 \times 10^{8}$ & $3.06 \times 10^{8}$ & $1.54 \times 10^{4}$ \\
\hline Reco. upgoing events & $6.08 \times 10^{7}$ & $2.98 \times 10^{7}$ & $1.24 \times 10^{4}$ \\
\hline Reco. upgoing events $+\beta<1^{\circ}$ & $3.90 \times 10^{7}$ & $1.57 \times 10^{7}$ & 360 \\
\hline Reco. upgoing $+\beta<1^{\circ}+\Lambda>-5.2$ & 3058 & 358 & 2408 \\
\hline
\end{tabular}

Notes. The last column shows the percentage of signal events assuming a neutrino flux proportional to an $E_{\nu}^{-2}$ spectrum.

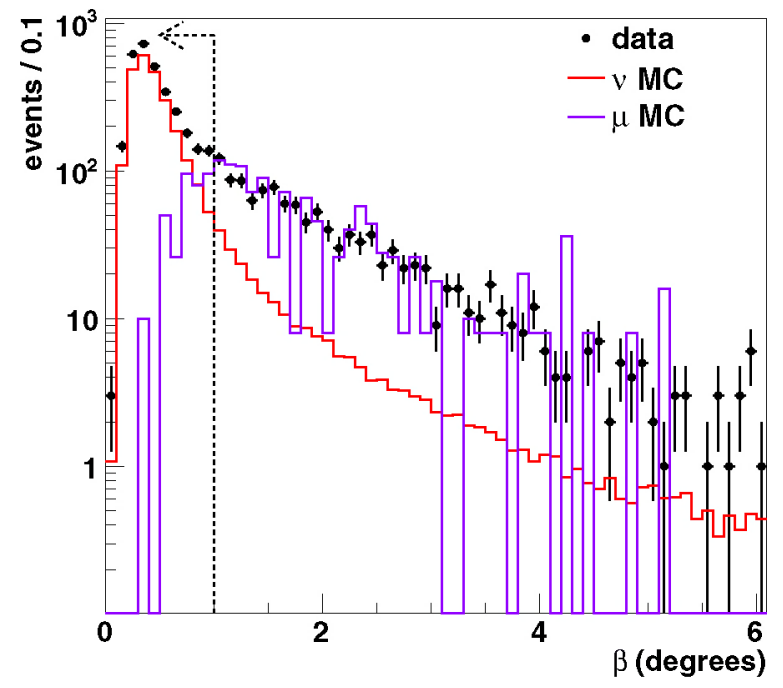

Figure 2. Distribution of the estimate of the error on the direction of the reconstructed upgoing muon track after applying a cut on the quality variable $\Lambda>-5.2$. The red line shows the Monte Carlo atmospheric neutrinos, the purple line the Monte Carlo misreconstructed atmospheric muons and the black dots the data. The vertical dashed line with the arrow shows where the selection cut is applied $\left(\beta<1^{\circ}\right)$.

(A color version of this figure is available in the online journal.)

misreconstructed as upgoing. In addition, assuming that the likelihood function near the fitted maximum follows a multivariate Gaussian distribution, the error on the zenith and azimuth angles are estimated from the covariance matrix. The angular uncertainty on the muon track direction, $\beta$, is obtained from these errors and can be used to further reject misreconstructed atmospheric muons as discussed in Section 6.

\section{EVENT SELECTION}

Neutrino candidates are selected requiring tracks reconstructed as upgoing and applying selection criteria. These criteria were chosen following a "blind" procedure, i.e., before performing the analysis on data. The effect of the selection cuts on data, expected background and signal efficiency are summarized in Table 1.

The estimated angular uncertainty on the muon track direction, $\beta$, is required to be smaller than $1 \mathrm{deg}$. This cut rejects $47 \%$ of the atmospheric muons which are misreconstructed as upgoing tracks.

To further reject atmospheric muons that were misreconstructed as upgoing, the quality variable $\Lambda$ is required to be larger than -5.2 . This value is chosen to optimize the discovery potential, i.e., the neutrino flux needed to have a $50 \%$ chance of discovering the signal at the $5 \sigma$ significance level assuming an $E_{v}^{-2}$ spectrum.

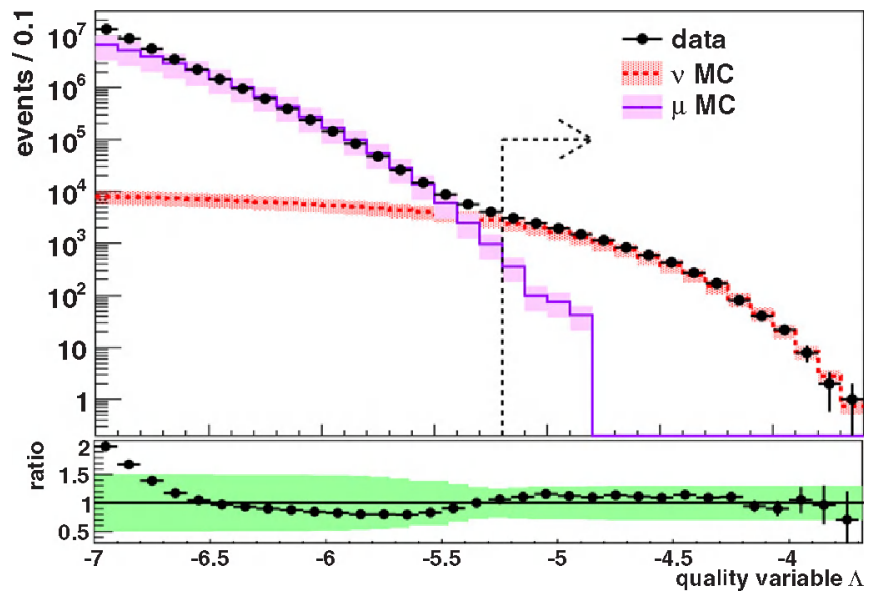

Figure 3. Cumulative distribution of the reconstruction quality variable $\Lambda$ for upgoing tracks which have an angular error estimate $\beta<1^{\circ}$. The bottom panel shows the ratio between data and simulations. The red line is for Monte Carlo atmospheric neutrinos, the purple line Monte Carlo misreconstructed atmospheric muons and the black dots the data. The vertical dashed line with the arrow shows where the selection cut is applied $(\Lambda>-5.2)$. The purple and red bands show the systematic uncertainties on the simulations as explained in Section 4 . The green band in the bottom panel shows the total contribution of these uncertainties.

(A color version of this figure is available in the online journal.)

Figure 2 shows the distribution of $\beta$ for upgoing events with $\Lambda>-5.2$. The cumulative distribution of $\Lambda$ for upgoing events is shown in Figure 3 . The cut on the angular error estimate $\beta$ is also applied. The excess of data compared to simulations at the lowest values of $\Lambda$ is due to a non-simulated contribution of events consisting of solely optical background. Figure 4 shows the distribution of the reconstructed cosine of the zenith angle for both data and simulation.

The final sample consists of 3058 neutrino candidate events out of a total of $\sim 4 \times 10^{8}$ triggered events. Simulations predict $358 \pm 179$ atmospheric muons and $2408 \pm 722$ atmospheric neutrinos, yielding a total expected events of $2766 \pm 743$. This is consistent with the observed rate within the quoted uncertainties (see Section 4).

\section{DETECTOR PERFORMANCE}

The response of the detector to a neutrino signal proportional to an $E_{v}^{-2}$ spectrum was obtained using the simulation described in Section 4 and applying the analysis cuts.

\subsection{Angular Resolution}

Figure 5 (left) shows the cumulative distribution of the angle between the direction of the reconstructed muon and that of the true neutrino. The median of this distribution is $0.46 \pm 0.10 \mathrm{deg}$. Of the selected events, $83 \%$ are reconstructed better than $1 \mathrm{deg}$. 


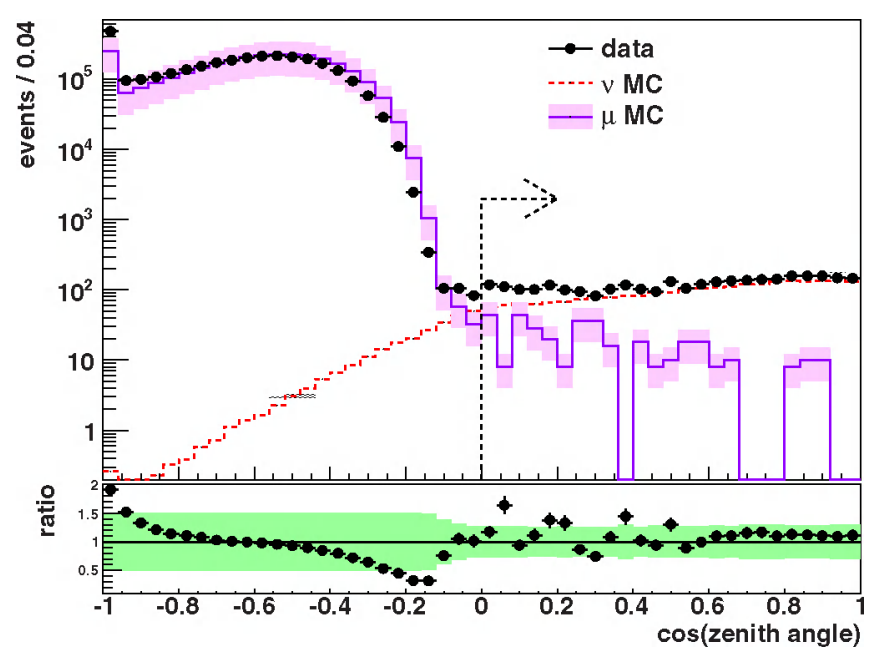

Figure 4. Distribution of the cosine of the zenith angle showing events with $\Lambda>-5.2$ and $\beta<1^{\circ}$. The bottom panel shows the ratio between data and the total contribution of simulations. The simulated distributions are shown for atmospheric muons and neutrinos. Systematic uncertainties on Monte Carlo atmospheric muons and neutrinos are shown by the purple and red bands, respectively. The green band corresponds to the sum of these uncertainties. The vertical dashed line with the arrow shows where the cut on the zenith angle is applied in order to select only upgoing events.

(A color version of this figure is available in the online journal.)

For the data sample in which the detector was operational with all 12 lines, the estimated angular resolution is $0.43 \pm 0.10 \mathrm{deg}$. The median of this angular error for the full data set considered in the analysis is shown in Figure 5 (right) as a function of the true neutrino energy.

The systematic uncertainty on the angular resolution quoted above has been estimated by varying the hit time resolution, $\Delta t$, in the simulation. Many possible effects can contribute to this resolution, including the PMT transit time spread, miscalibrations of the timing system and possible spatial misalignments of the detector. The hit time resolution directly impacts both the angular resolution and the number of events passing the quality criteria. Simulations using different $\Delta t$ values were compared with data in order to determine the best agreement in the lambda distribution. and was obtained for $\Delta t=2.5 \mathrm{~ns}$. This can be compared to the TTS of the PMT, which is $1.3 \mathrm{~ns}$ (standard deviation). However, the PMT time response is not Gaussian and the degraded resolution was found to partly account for the tails. A $\Delta t$ of $2.5 \mathrm{~ns}$ yields the quoted angular resolution of 0.46 and is the value used in the plots shown in this paper.

For $\Delta t=3.4 \mathrm{~ns}$, the simulations show a deterioration in angular resolution of $30 \%$ and the number of selected neutrino events in the data exceeds the simulated neutrino signal by $2 \sigma$, where $\sigma$ refers to the uncertainty on the atmospheric neutrino flux model. Hence, this value of $\Delta t$ is excluded from the data. Assuming a linear dependency, this argument translates to a ( $1 \sigma$ ) uncertainty on the angular resolution of $\sim 15 \%$.

The absolute orientation of the detector is known with an accuracy of $\sim 0.1$ (Adrián-Martínez et al. 2012).

\subsection{Acceptance}

The neutrino effective area, $A_{v}^{\text {eff }}$, is defined as the ratio between the neutrino event rate, $R_{v}\left(E_{v}\right)$, and the cosmic neutrino flux, $\Phi\left(E_{\nu}\right)$. The flux is assumed to consist of equal amounts of $v_{\mu}$ and $\bar{v}_{\mu}$. The neutrino effective area depends on the neutrino cross section, the absorption of neutrinos through the Earth and the muon detection (and selection) efficiency. It can be
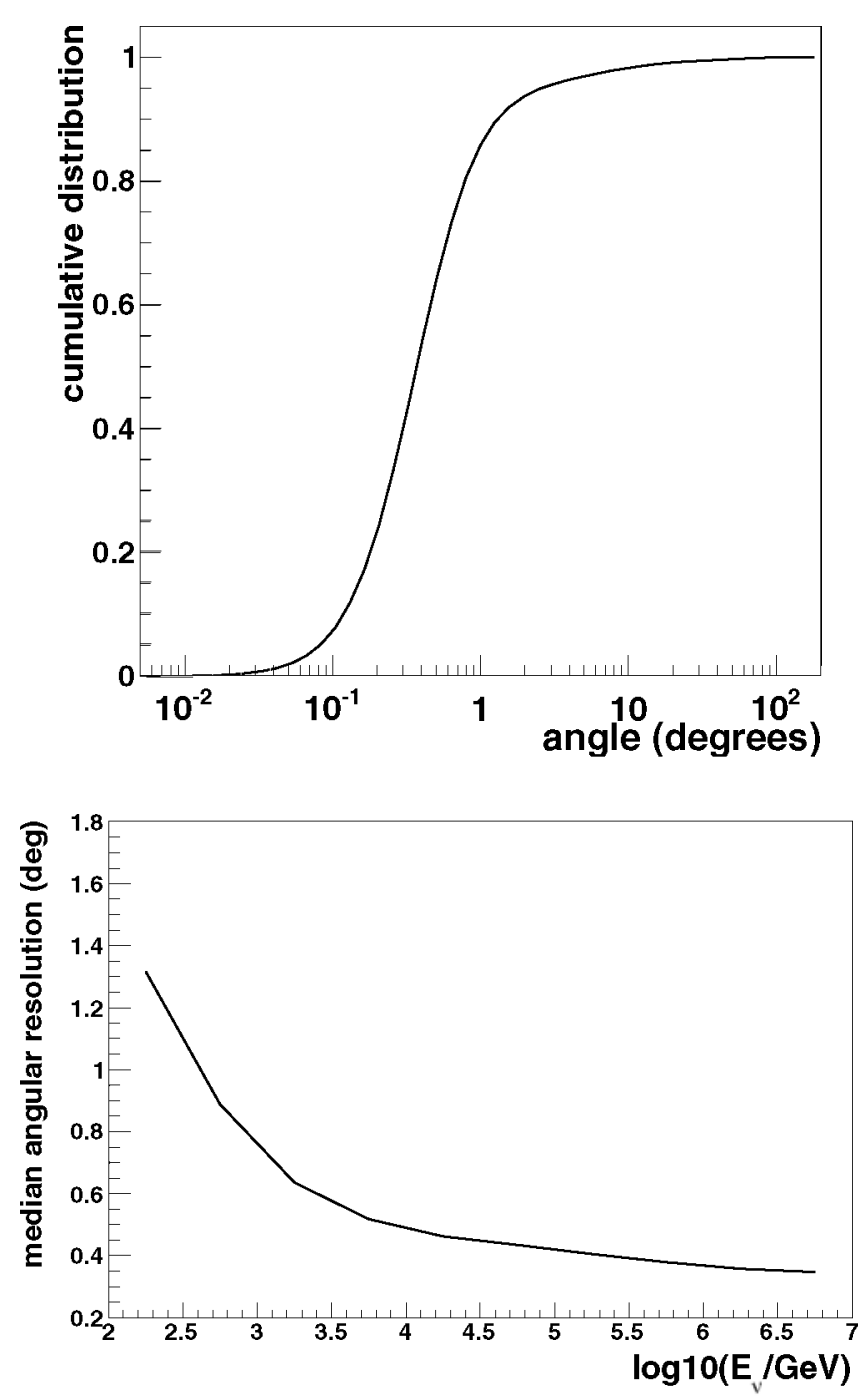

Figure 5. Top: cumulative distribution of the angle between the reconstucted muon direction and the true neutrino direction for upgoing events of the whole data set. A neutrino spectrum proportional to $E_{v}^{-2}$ is assumed. Bottom: the median angle as a function of the neutrino energy $E_{\nu}$. In these plots the cuts $\Lambda>-5.2$ and $\beta<1^{\circ}$ are applied.

considered as the equivalent area of a $100 \%$ efficient detector. Figure 6 shows the effective area as a function of the neutrino energy and declination, $\delta_{v}$.

The analysis is primarily concerned with cosmic sources emitting neutrinos with an $E_{v}^{-2}$ power law of the form

$$
\frac{d N_{\nu}}{d E_{\nu} d t d S}=\phi \times\left(\frac{E_{\nu}}{\mathrm{GeV}}\right)^{-2} \mathrm{GeV}^{-1} \mathrm{~cm}^{-2} \mathrm{~s}^{-1},
$$

where the constant $\phi$ is the flux normalization. The acceptance, $A\left(\delta_{\nu}\right)$, for such a flux, is defined as the constant of proportionality between $\phi$ and the expected number of events in the source direction and can be expressed in terms of the effective area as

$$
A\left(\delta_{\nu}\right)=\phi^{-1} \iint d t d E_{\nu} A_{\nu}^{\mathrm{eff}}\left(E_{\nu}, \delta_{\nu}\right) \frac{d N_{\nu}}{d E_{\nu}, d t} .
$$

The acceptance for this analysis is shown in Figure 6 (bottom). For a source at a declination of $-90(0)^{\circ}, A=8.8(4.8) \times 10^{7}$ $\mathrm{GeV}^{-1} \mathrm{~cm}^{2} \mathrm{~s}$ which means that a total of $8.8(4.8)$ neutrino candidates would be selected from a point-like source emitting a flux of $10^{-7} \times\left(E_{\nu} / \mathrm{GeV}\right)^{-2} \mathrm{GeV}^{-1} \mathrm{~cm}^{-2} \mathrm{~s}^{-1}$. 

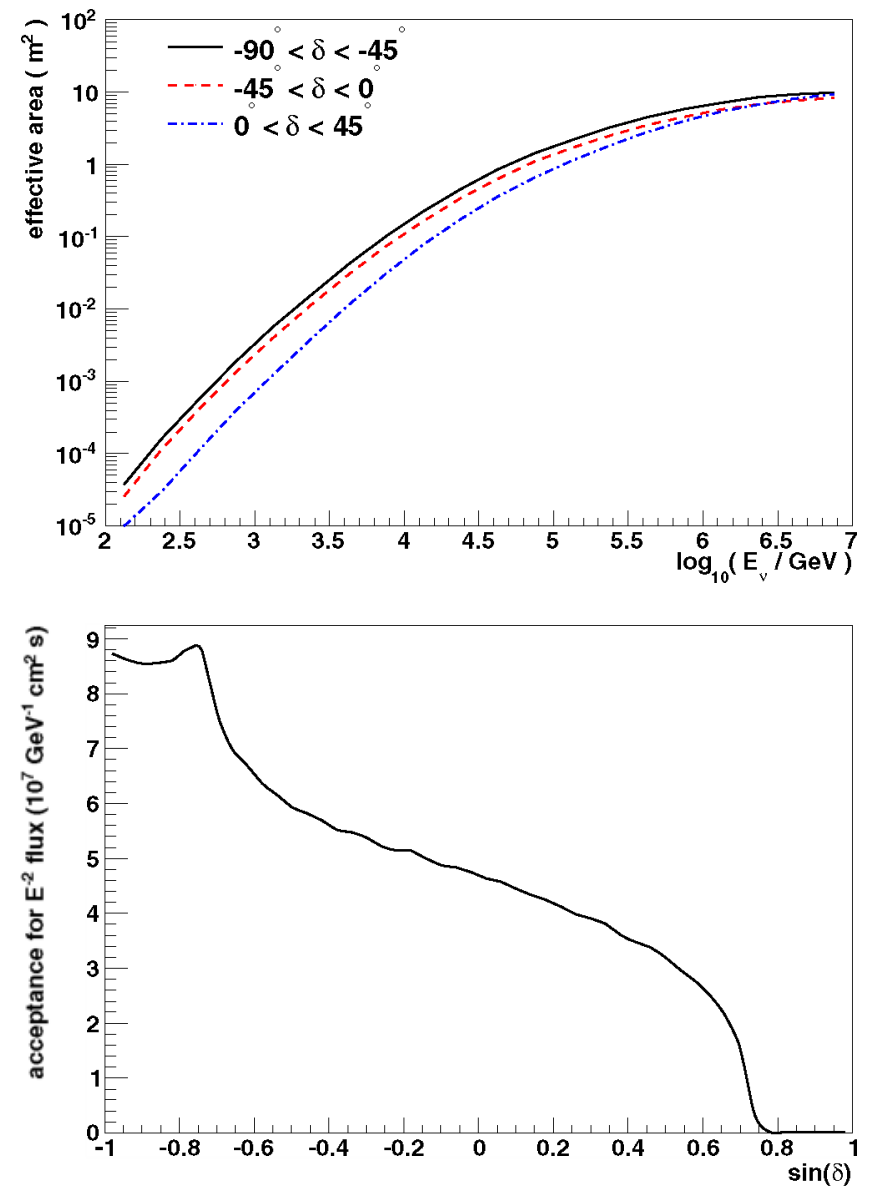

Figure 6. Top: the neutrino effective area $A_{v}^{\text {eff }}$ for the selected events as a function of the neutrino energy $E_{\nu}$ for three different declination bands. Bottom: acceptance of the detector which is proportional to the number of events that would be detected and selected from a point-like source at a given declination assuming a flux of $10^{-7} \times\left(E_{\nu} / \mathrm{GeV}\right)^{-2} \mathrm{GeV}^{-1} \mathrm{~cm}^{-2} \mathrm{~s}^{-1}$ as a function of the sine of the declination.

(A color version of this figure is available in the online journal.)

To constrain the systematic uncertainty on the acceptance, a comparison between the atmospheric neutrino data and a simulation was performed in which the efficiency of each of the OMs was reduced by $15 \%$, which yields a $12 \%$ reduction of the signal events for an $E_{v}^{-2}$ flux. The atmospheric neutrino yield would instead be reduced by $40 \%$ to be compared to the $30 \%$ error on its flux normalization. Therefore, the $15 \%$ uncertainty on the acceptance can be therefore considered a conservative choice.

\section{SEARCH METHOD}

Two alternative searches for point-like neutrino sources have been performed. The full-sky search looks for an excess of signal events over the atmospheric muon and neutrino background anywhere in the visible sky, i.e., in the declination range $\left(-90^{\circ},+48^{\circ}\right)$. In the candidate list search, the presence of an excess of events is tested at the locations of the 51 predefined candidate sources given in Table 2. They include the 24 source candidates from Adrián-Martínez et al. (2011) and 27 new sources selected considering their gamma-ray flux and their visibility at the ANTARES site as the selection criteria. Among the Galactic sources only TeV gamma-ray emitters are considered. No such requirement is imposed for

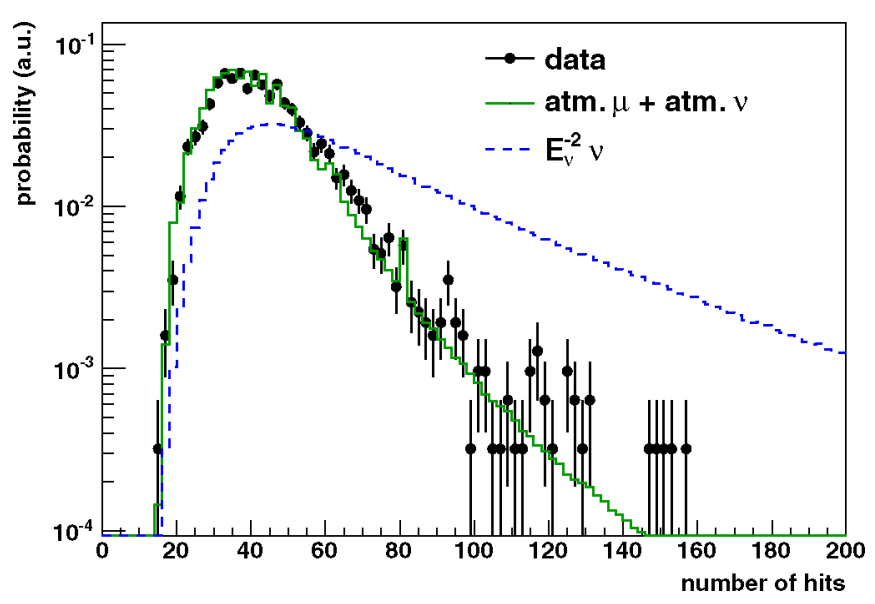

Figure 7. Distribution of the number of hits used in the reconstruction, for the selected data (black dots), and the total Monte Carlo background contribution, i.e., atmospheric muons and atmospheric neutrinos (solid green line). The dashed blue line corresponds to the cosmic neutrino signal assuming an $E_{v}^{-2}$ spectrum. The distribution is normalized to the integral of the total number of events. All the cuts described in Section 6 are applied.

(A color version of this figure is available in the online journal.)

extragalactic sources as $\mathrm{TeV}$ gamma rays may be absorbed by the extragalactic background light (Nikishov 1962; Gould et al. 1966; Jelley 1966).

The algorithm for the cluster search uses an unbinned maximum likelihood (Barlow 1990) which is defined as

$$
\begin{aligned}
\log \mathcal{L}_{\mathrm{s}+\mathrm{b}}= & \sum_{i} \log \left[\mu_{\mathrm{s}} \times \mathcal{F}\left(\psi_{i}\left(\alpha_{\mathrm{s}}, \delta_{\mathrm{s}}\right)\right) \times \mathcal{N}^{\mathrm{s}}\left(N_{\text {hits }}^{i}\right)\right. \\
& \left.+\mathcal{B}\left(\delta_{i}\right) \times \mathcal{N}^{\mathrm{bg}}\left(N_{\text {hits }}^{i}\right)\right]-\mu_{\mathrm{s}}-\mu_{\mathrm{bg}},
\end{aligned}
$$

where the sum is over the events; $\mathcal{F}$ is a parameterization of the point spread function, i.e., the probability density function of reconstructing event $i$ at an angular distance $\psi_{i}$ from the true source location $\left(\alpha_{\mathrm{s}}, \delta_{\mathrm{s}}\right) ; \mathcal{B}$ is a parameterization of the background rate obtained from the distribution of the observed declination of the 3058 selected events; $\mu_{\mathrm{s}}$ and $\mu_{\mathrm{bg}}$ are the mean number of signal events and the total number of expected background events; $N_{\text {hits }}^{i}$ is the number of hits used in the reconstruction. $\mathcal{N}^{\mathrm{s}}\left(N_{\text {hits }}^{i}\right)$ and $\mathcal{N}^{\mathrm{bg}}\left(N_{\text {hits }}^{i}\right)$ are the probabilities of measuring $N_{\text {hits }}^{i}$ hits for signal and background, respectively. The distribution of $N_{\text {hits }}^{i}$ for data and Monte Carlo events is shown in Figure 7. Figure 8 shows the distribution of $N_{\text {hits }}$ for signal as a function of the true neutrino energy.

In the candidate list search, the sum in Equation (4) incorporates the events located in a cluster within $20 \mathrm{deg}$ of the source. Events further away have $\mathcal{F} \simeq 0$ and thus contribute a constant factor to the likelihood. In the full-sky search potentially significant clusters are first identified by selecting at least four events within a cone of $3 \mathrm{deg}$ diameter. Using a larger diameter or a bigger/lower number of required events increases the computation time without a significant improvement in the sensitivity.

In the candidate list search the likelihood is maximized by numerically fitting the mean number of signal events, $\mu_{s}$, with the source location fixed. In the full-sky search the likelihood maximization yields the source coordinates and $\mu_{s}$ for each cluster. After likelihood maximization a test statistic, $\mathcal{Q}$, is computed:

$$
\mathcal{Q}=\log \mathcal{L}_{\mathrm{s}+\mathrm{b}}^{\max }-\log \mathcal{L}_{\mathrm{b}}
$$

where $\log \mathcal{L}_{\mathrm{s}+\mathrm{b}}^{\max }$ is the maximum value of the likelihood provided by the fit and $\log \mathcal{L}_{\mathrm{b}}$ is the likelihood computed for the 
Table 2

Results from the Search for High-energy Neutrinos from Sources in the Candidate List

\begin{tabular}{|c|c|c|c|c|c|c|c|c|c|}
\hline Source Name & $\begin{array}{l}\alpha_{s} \\
\left(^{\circ}\right)\end{array}$ & $\begin{array}{c}\delta_{s} \\
\left(^{\circ}\right)\end{array}$ & $p$ & $\phi^{90 \% \mathrm{CL}}$ & Source Name & $\begin{array}{l}\alpha_{s} \\
\left(^{\circ}\right)\end{array}$ & $\begin{array}{c}\delta_{S} \\
\left(^{\circ}\right)\end{array}$ & $p$ & $\phi^{90 \% \mathrm{CL}}$ \\
\hline HESS J1023-575 & 155.83 & -57.76 & 0.41 & 6.6 & SS 433 & -72.04 & 4.98 & $\cdots$ & 4.6 \\
\hline $3 \mathrm{C} 279$ & -165.95 & -5.79 & 0.48 & 10.1 & HESS J1614-518 & -116.42 & -51.82 & $\cdots$ & 2.0 \\
\hline GX 339-4 & -104.30 & -48.79 & 0.72 & 5.8 & RX J1713.7-3946 & -101.75 & -39.75 & $\cdots$ & 2.7 \\
\hline Cir X-1 & -129.83 & -57.17 & 0.79 & 5.8 & $3 \mathrm{C} 454.3$ & -16.50 & 16.15 & $\cdots$ & 5.5 \\
\hline MGRO J1908+06 & -73.01 & 6.27 & 0.82 & 10.1 & W28 & -89.57 & -23.34 & $\cdots$ & 3.4 \\
\hline ESO 139-G12 & -95.59 & -59.94 & 0.94 & 5.4 & HESS J0632+057 & 98.24 & 5.81 & $\cdots$ & 4.6 \\
\hline HESS J1356-645 & -151.00 & -64.50 & 0.98 & 5.1 & PKS 2155-304 & -30.28 & -30.22 & $\cdots$ & 2.7 \\
\hline PKS 0548-322 & 87.67 & -32.27 & 0.99 & 7.1 & HESS J1741-302 & -94.75 & -30.20 & $\cdots$ & 2.7 \\
\hline HESS J1837-069 & -80.59 & -6.95 & 0.99 & 8.0 & Centaurus A & -158.64 & -43.02 & $\cdots$ & 2.1 \\
\hline PKS 0454-234 & 74.27 & -23.43 & $\cdots$ & 7.0 & RX J0852.0-4622 & 133.00 & -46.37 & $\cdots$ & 1.5 \\
\hline IceCube hotspot & 75.45 & -18.15 & $\cdots$ & 7.0 & 1ES $1101-232$ & 165.91 & -23.49 & $\cdots$ & 2.8 \\
\hline PKS 1454-354 & -135.64 & -35.67 & $\cdots$ & 5.0 & Vela X & 128.75 & -45.60 & $\cdots$ & 1.5 \\
\hline RGB J0152+017 & 28.17 & 1.79 & $\cdots$ & 6.3 & W51C & -69.25 & 14.19 & $\cdots$ & 3.6 \\
\hline Geminga & 98.31 & 17.01 & $\cdots$ & 7.3 & PKS 0426-380 & 67.17 & -37.93 & $\ldots$ & 1.4 \\
\hline PSR B1259-63 & -164.30 & -63.83 & $\cdots$ & 3.0 & LS 5039 & -83.44 & -14.83 & $\ldots$ & 2.7 \\
\hline PKS 2005-489 & -57.63 & -48.82 & $\cdots$ & 2.8 & W44 & -75.96 & 1.38 & $\cdots$ & 3.1 \\
\hline HESS J1616-508 & -116.03 & -50.97 & $\cdots$ & 2.7 & RCW 86 & -139.32 & -62.48 & $\cdots$ & 1.1 \\
\hline HESS J1503-582 & -133.54 & -58.74 & $\cdots$ & 2.8 & Crab & 83.63 & 22.01 & $\cdots$ & 4.1 \\
\hline HESS J1632-478 & -111.96 & -47.82 & $\cdots$ & 2.6 & HESS J1507-622 & -133.28 & -62.34 & $\cdots$ & 1.1 \\
\hline H $2356-309$ & -0.22 & -30.63 & $\cdots$ & 3.9 & 1ES 0347-121 & 57.35 & -11.99 & $\cdots$ & 1.9 \\
\hline MSH $15-52$ & -131.47 & -59.16 & $\cdots$ & 2.6 & VER J0648+152 & 102.20 & 15.27 & $\cdots$ & 2.8 \\
\hline Galactic Center & -93.58 & -29.01 & $\cdots$ & 3.8 & PKS 0537-441 & 84.71 & -44.08 & $\cdots$ & 1.3 \\
\hline HESS J1303-631 & -164.23 & -63.20 & $\cdots$ & 2.4 & HESS J1912+101 & -71.79 & 10.15 & $\cdots$ & 2.5 \\
\hline HESS J1834-087 & -81.31 & -8.76 & $\cdots$ & 4.3 & PKS 0235+164 & 39.66 & 16.61 & $\cdots$ & 2.8 \\
\hline \multirow[t]{2}{*}{ PKS $1502+106$} & -133.90 & 10.52 & $\cdots$ & 5.2 & IC443 & 94.21 & 22.51 & $\ldots$ & 2.8 \\
\hline & & & & & PKS 0727-11 & 112.58 & 11.70 & $\ldots$ & 1.9 \\
\hline
\end{tabular}

Notes. The equatorial coordinates $\left(\alpha_{\mathrm{s}}, \delta_{\mathrm{s}}\right)$ in degrees, $p$-value $(p)$ probability and the $90 \% \mathrm{CL}$ upper limit on the $E_{\nu}^{-2}$ flux intensity $\phi^{90 \% \mathrm{CL}}$ in units of $10^{-8} \mathrm{GeV}^{-1} \mathrm{~cm}^{-2} \mathrm{~s}^{-1}$ are given (sorted in order of increasing $p$-value) for the 51 selected sources.

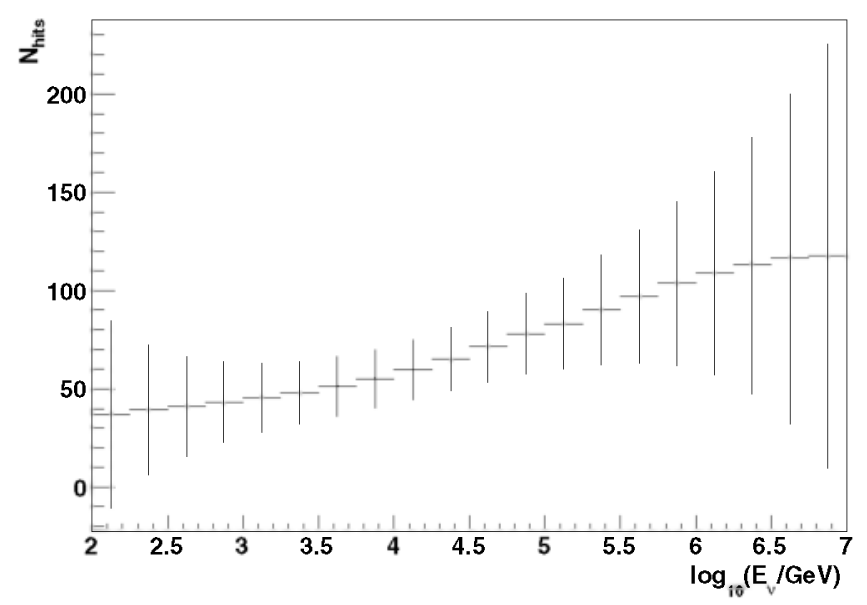

Figure 8. Distribution of the mean of number of hits, $\left\langle N_{\text {hits }}\right\rangle$, as a function of $E_{\nu}$ for Monte Carlo signal events assuming a neutrino spectrum proportional to $E_{v}^{-2}$. The error bars represent the standard deviation of the $N_{\text {hits }}$ distribution. The final cuts of the analysis described in Section 6 are included.

background only case $\left(\mu_{s}=0\right)$. A large value of $\mathcal{Q}$ indicates a better compatibility with the signal hypothesis. In case of a full-sky search only the cluster with the largest value of the test statistic is considered.

\section{PSEUDO-EXPERIMENT GENERATION AND LIMIT SETTING}

In order to evaluate the sensitivity of the analysis, pseudoexperiments are generated simulating background and signal. Background events are randomly generated by sampling the declination from the parameterization $\mathcal{B}$. The right ascension is sampled from a uniform distribution. The simulation of the signal is performed by adding events around the coordinates of the source, sampling in this case the angular distance $\psi_{i}$ of the event $i$ to the source location from a three-dimensional distribution of the reconstruction error as a function of the declination and the number of hits. At this stage the systematic uncertainties on the angular resolution and on the absolute orientation of the detector are included by varying the simulated parameters of the events of each experiment, such as the zenith and azimuth angles, with the required uncertainty. An example of the distribution of the test statistic $\mathcal{Q}$ obtained by performing the search on a large number of pseudo-experiments for the full-sky search is shown in Figure 9 for the background only hypothesis and for experiments where several signal events are added to the background at a declination $\delta=-70^{\circ}$. Figure 9 also shows the values of $\mathcal{Q}$ corresponding to $p$-values of $2.7 \times$ $10^{-3}$ and $5.7 \times 10^{-7}$, i.e., $3 \sigma$ and $5 \sigma$. To compute the latter value, the distribution of the test statistic for the background only hypothesis has been extrapolated using an exponential fit.

The sensitivity and the flux upper limits at $90 \%$ confidence level (CL) are computed following the Feldman \& Cousins prescription (Feldman \& Cousins 1998). The systematic uncertainty on the acceptance is taken into account in the computation.

\section{DISCOVERY POTENTIAL}

Figure 10 shows the probability of making a discovery at the $3 \sigma$ and $5 \sigma$ significance level, as a function of the mean number of signal events. The same curves are shown for a search which does not use $N_{\text {hits }}$ in the likelihood. The inclusion of the $N_{\text {hits }}$ pdfs in the likelihood function reduces the number of events (and therefore the signal flux) needed for a discovery by $\sim 25 \%$. 


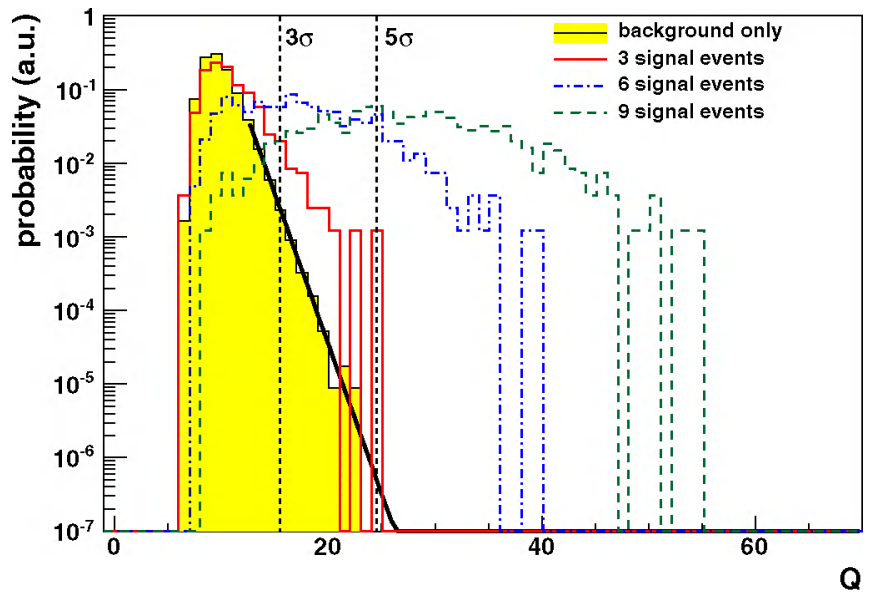

Figure 9. Distribution of the test statistic $\mathcal{Q}$ for the full-sky search. The full yellow histogram is for the background only experiments. The red, blue, and green lines are for 3,6 , and 9 signal events generated from a source with an $E_{v}^{-2}$ spectrum at a declination of $-70^{\circ}$. The vertical dotted lines show the values of $\mathcal{Q}$ corresponding to the $3 \sigma$ and $5 \sigma$ significance level.

(A color version of this figure is available in the online journal.)

The worsening of the $3 \sigma$ and $5 \sigma$ discovery probability for a neutrino flux model with an exponential cutoff parameterized as $d N / d E=\phi \times\left(E_{v} / \mathrm{GeV}\right)^{-2} \exp \left(-E_{\nu} / E_{\mathrm{c}}\right)$, with $E_{\mathrm{c}}$ the cutoff energy, was estimated. In this case, for a source at a declination of $\delta=-70^{\circ}$, the mean number of signal events needed for a $5 \sigma$ discovery assuming a cutoff energy $E_{\mathrm{c}}=1 \mathrm{TeV}$ is a factor 2 higher compared to that without an exponential cutoff.

Simulations show that for a source with Gaussian extension $\sigma_{\text {source }}=1^{\circ}$ at a declination of $\delta=-70^{\circ}$, the flux needed to claim a $5 \sigma$ discovery is a factor 1.2 higher compared to a point-like source.

\section{RESULTS}

A map in equatorial coordinates of the pre-trial significances of every point in the sky that is visible below the horizon at the ANTARES site is shown in Figure 11.

In the full-sky search the most significant cluster is located at $(\alpha, \delta)=(-46.5,-65.0)$, where $5(9)$ events are within $1(3)$ deg of this position. For this cluster the fit assigns 5.1 as signal events, and the value of the test statistic is $\mathcal{Q}=13.1$. The corresponding $p$-value is obtained by comparing the value of the observed test statistic $\mathcal{Q}$ with the simulated $\mathcal{Q}$ distribution for the background only hypothesis. The post-trial $p$-value is $2.6 \%$, which is equivalent to $2.2 \sigma$ (using the two-sided convention).

The results from the search up the 51 a priori selected candidate sources are presented in Table 2 and shown in Figure 12. The most signal-like candidate source in the list is HESS J1023-575. The maximum likelihood fit yield $\mu_{s}=2.0$ and the test statistic value is $\mathcal{Q}=2.4$. The post-trial $p$-value of this cluster is $41 \%$, compatible with a background fluctuation. Since no statistically significant cluster of events has been found, upper limits (Feldman \& Cousins at $90 \%$ CL) for an $E_{v}^{-2}$ flux are obtained for the candidate list sources. These limits and the corresponding 90\% CL sensitivity are plotted in Figure 12 as a function of the source declination. Also indicated are the published limits from other experiments.

The values of the observed limits are distributed around the expected median limit (i.e., the sensitivity) as expected. As the analysis uses only upgoing events, the declination dependence of the sensitivity is mostly dictated by the fraction of time an

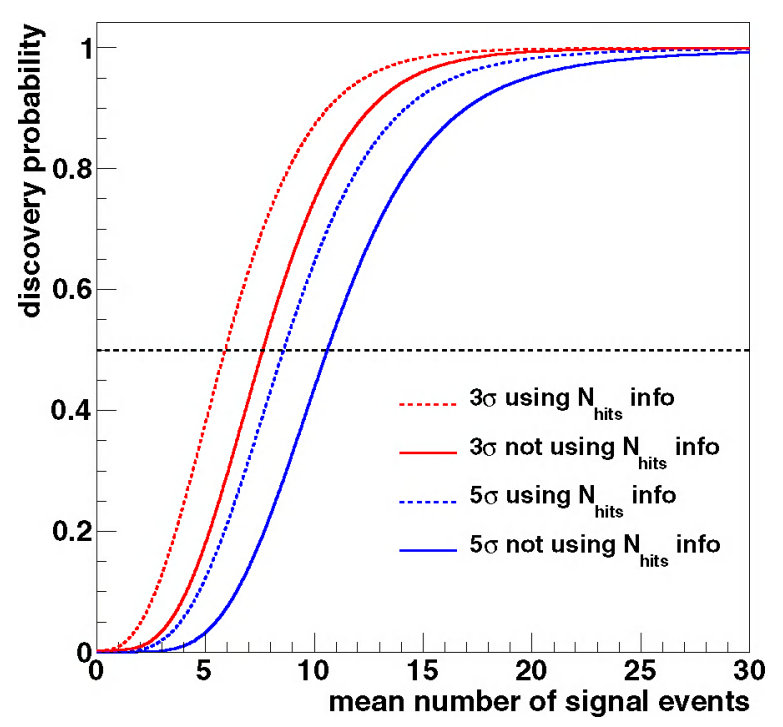

Figure 10. Probability for a $3 \sigma$ (red lines) and $5 \sigma$ (blue lines) full-sky search discovery as a function of the mean number of signal events from a source at $\delta=-70^{\circ}$ with a neutrino spectrum proportional to $E_{\nu}^{-2}$. The dotted blue and red lines are for the likelihood described; the solid lines refer to the case where $N_{\text {hits }}$ is not used. The horizontal dotted black line corresponds to the probability to make a discovery in $50 \%$ of the pseudo-experiments.

(A color version of this figure is available in the online journal.)

object is below the horizon. At the geographical location of ANTARES, the part of the sky with $\delta>42^{\circ}$ is always above the horizon, hence limits are not derived in that region. The IceCube analysis (Abbasi et al. 2011) shown in Figure 12 includes very high energy downgoing events and therefore quotes limits in part of the Southern Hemisphere.

\subsection{Limits for Specific Models}

Measurements of $\mathrm{TeV}$ gamma rays from the H.E.S.S. (Aharonian et al. 2006, 2007) telescope may indicate a possible hadronic scenario for the shell-type supernova remnant RX J1713.7-3946 and the pulsar wind nebula Vela X. The first observation of RX J1713.7-3946 with the Fermi Large Area Telescope (Abdo et al. 2011) shows that the gamma-ray emission seems to be compatible with a leptonic origin. However, composite models are also possible as discussed in Zirakashvili \& Aharonian (2010).

In Kappes et al. (2007) the neutrino flux and signal rates are estimated for these objects using the energy spectrum measured by H.E.S.S and by approximating the source extension with a Gaussian distribution. The spectrum for these models is shown in Figure 13. Assuming these models, and taking into account the measured source extension, 90\% CL upper limits on the flux normalization were computed for both sources. The model rejection factor (MRF; Hill \& Rawlins 2003), i.e., the ratio between the $90 \%$ CL upper limit and the expected number of signal events, is also calculated. Figure 13 summarizes these results. For RX J1713.7-3946 the upper limit is a factor 8.8 higher than the theoretical prediction. For Vela X the upper limit is a factor 9.1 higher than the model. In both cases these are the most restrictive limits for the emission models considered.

\section{CROSS-CHECK WITH THE EXPECTATTON-MAXIMIZATION METHOD}

The results discussed in the previous section have been crosschecked using the expectation-maximization (E-M) algorithm 


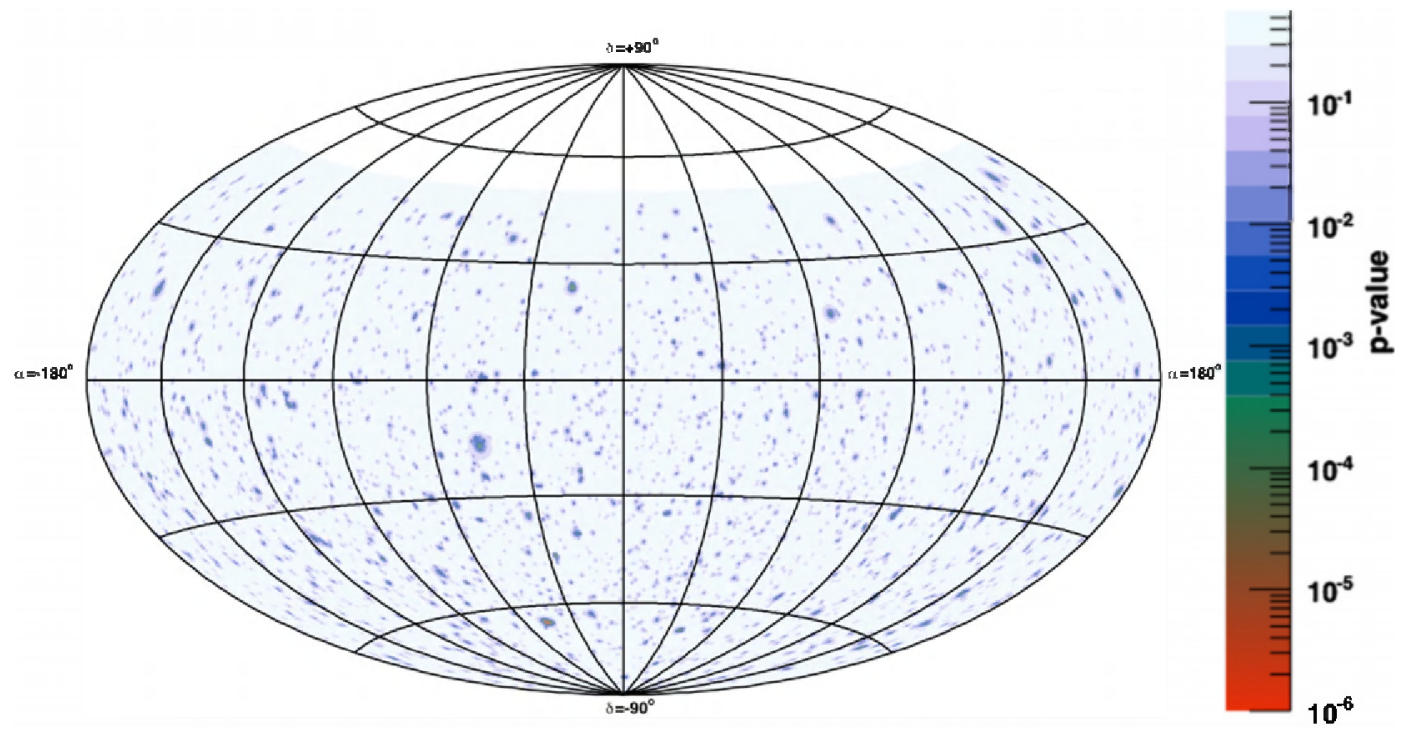

Figure 11. Sky map in equatorial coordinates showing the $p$-values obtained for the point-like clusters evaluated in the full-sky scan; the penalty factor accounting for the number of trials is not considered in this calculation.

(A color version of this figure is available in the online journal.)

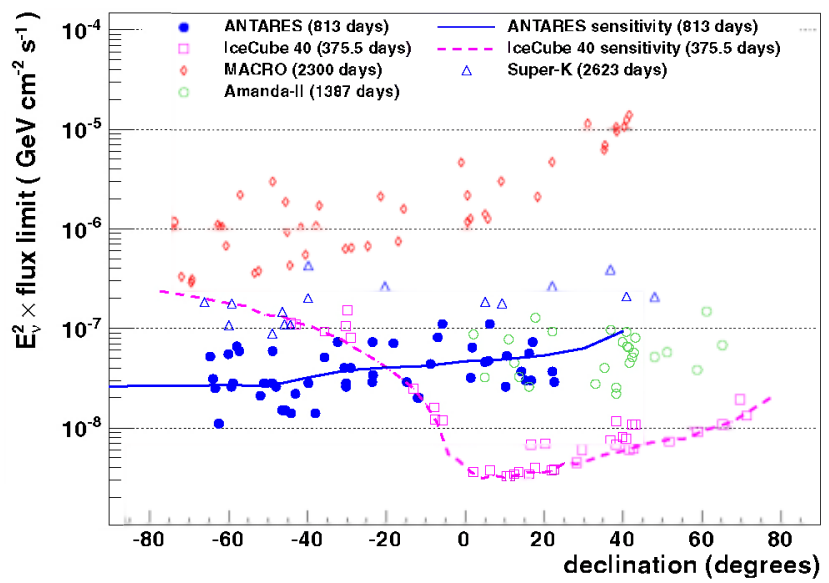

Figure 12. Limits set on the $E_{v}^{-2}$ flux for the 51 sources in the candidate list (see Table 2). Upper limits, previously reported by other neutrino experiments, on sources from both Northern and Southern sky are also included (Ambrosio et al. 2001; Thrane et al. 2009; Abbasi et al. 2009). The ANTARES sensitivity of this analysis is shown as a solid line and the IceCube 40 sensitivity as a dashed line (Abbasi et al. 2011).

(A color version of this figure is available in the online journal.)

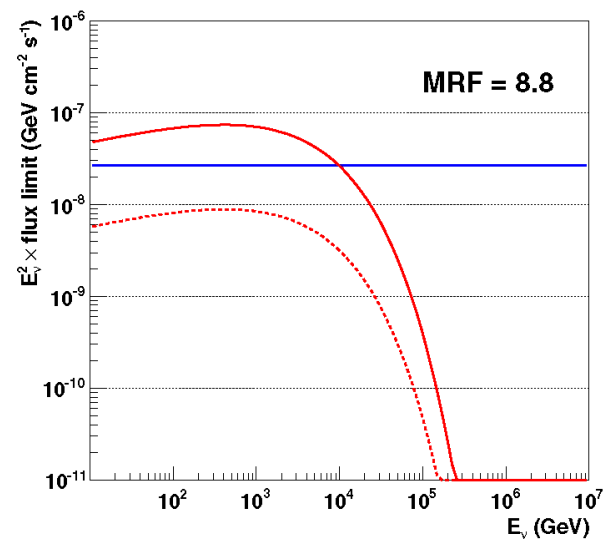

applied to the problem of the search for point sources (Dempster et al. 1977; Aguilar et al. 2008). The E-M method is an iterative approach to maximum likelihood estimations of finite mixture problems, which are described by different probability density functions. In the case of a search for point sources the mixture model can be expressed as the sum of two components:

$$
\begin{aligned}
\log \mathcal{L}_{\mathrm{s}+\mathrm{b}}= & \sum_{i} \log \left[\left(\frac{\mu_{\mathrm{s}}}{\mu_{\mathrm{t}}}\right) \times \mathcal{F}\left(\psi_{\mathrm{i}}\left(\alpha_{\mathrm{s}}, \delta_{\mathrm{s}}\right)\right) \times \frac{\mathcal{N}^{\mathrm{s}}\left(\mathrm{N}_{\text {hits }}^{\mathrm{i}}\right)}{\mathcal{N}^{\mathrm{b} \xi}\left(\mathrm{NN}_{\text {hits }}^{\mathrm{i}}\right)}\right. \\
& \left.+\left(1-\frac{\mu_{\mathrm{s}}}{\mu_{\mathrm{t}}}\right) \times \mathcal{D}\left(\delta_{i}\right)\right]
\end{aligned}
$$

where the signal pdf $(\mathcal{F})$ is modeled as a two-dimensional Gaussian, and $\mathcal{D}$ is a polynomial parameterization of the probability distribution of the events in declination; as in Equation (4), the sum runs over all the events in the data set, $\mu_{t}$, and the number of hits is used to better discriminate between background-like and signal-like events.

In comparison with the previous search method, the E-M algorithm uses a different likelihood description of the events

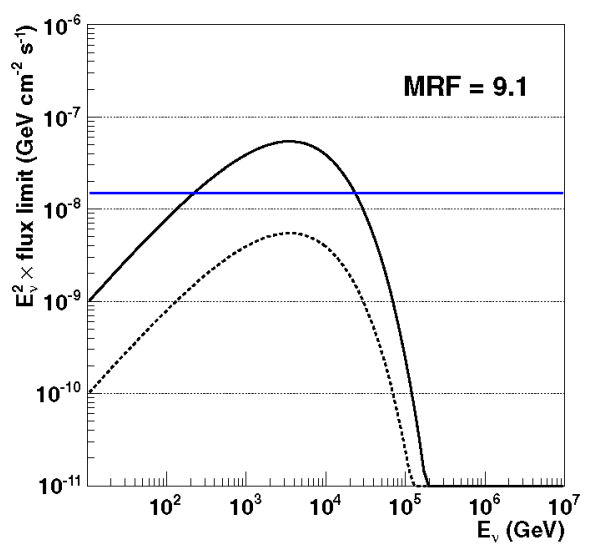

Figure 13. Neutrino flux models (dashed lines) and 90\% CL upper limit (solid lines) for RX J1713.7-3946 (left) and Vela X (right). Also shown are the $E^{-2}$ point-source limit (solid horizontal blue lines) presented in Table 2. The models are taken from Kappes et al. (2007).

(A color version of this figure is available in the online journal.) 
and follows an analytical optimization procedure that consists of two steps. In the expectation step the log-likelihood is evaluated using the current set of parameters describing the source properties. Then, during the maximization step, a new set of parameters is computed maximizing the expected loglikelihood. These parameters are the number of signal events attributed to the source, the source coordinates (in the full-sky search) and the standard deviations of the Gaussian describing the signal pdf. In this sense the E-M method has the freedom to adapt itself to the extension of the source. The test statistic used to determine the significance of a potential point source is obtained as in Equation (5).

\subsection{Results}

The most signal-like cluster found in the full-sky search is the same as that found by the search method described in Section 8 . The number of signal events estimated by the algorithm is $\mu_{\mathrm{s}}=5.3$. The observed value of the test statistic, $\mathcal{Q}=12.8$, or a larger one occurs in $p=2.6 \%$ of the background-only experiments. No significant excess of events was found in the location of any of the candidate list sources. The lowest $p$-value is 0.87 (post-trial corrected) and corresponds to the position of $3 \mathrm{C}-279$. The results obtained with the two search methods described above are consistent.

\section{CONCLUSIONS}

The results of a search for cosmic neutrino point sources with the ANTARES telescope using data taken in 2007-2010 have been presented. A likelihood ratio method has been used to search for clusters of neutrinos in the sky map. In addition to the position of the reconstructed events, the information of the number of hits has been used as an estimate of the neutrino energy. This improves the discrimination between the cosmic signal and the background of atmospheric neutrinos.

Two searches have been performed: within a list of candidate sources and in the whole sky. No statistically significant excess has been found in either case. In the full-sky search, the most signal-like cluster is at $(\alpha, \delta)=(-46.5,-65.0)$. It consists of nine events inside a $3 \mathrm{deg}$ cone, to which the likelihood fit assigns 5.1 signal events. The corresponding $p$-value is 0.026 with a significance of $2.2 \sigma$ (two-sided). The most significant excess in the candidate list search corresponds to HESS J1023-575, with a post-trial $p$-value of $0.41 .90 \%$ CL Upper limits on the neutrino flux normalization are set at $1-10 \times 10^{-8} \mathrm{GeV} \mathrm{cm}^{-2} \mathrm{~s}^{-1}$ in the range from 4 to $700 \mathrm{TeV}$ ( $80 \%$ of the signal), assuming an energy spectrum of $E_{v}^{-2}$, and are the most restrictive ones for a large part of the Southern sky. These limits are a factor $\sim 2.7$ better than those obtained in the previous ANTARES analysis based on the 2007-2008 data. Limits for specific models of RX J1713.7-3946 and Vela X, which include information on the source morphology and spectrum, were also given, resulting in a factor $\sim 9$ above the predicted fluxes.

The authors acknowledge the financial support of the funding agencies: Centre National de la Recherche Scientifique (CNRS), Commissariat à l'énergie atomique et aux énergies alternatives (CEA), Commission Européenne (FEDER fund and Marie Curie Program), Région Alsace (contrat CPER), Région Provence-Alpes-Côte d'Azur, Département du Var and Ville de La Seyne-sur-Mer, France; Bundesministerium für Bildung und Forschung (BMBF), Germany; Istituto Nazionale di Fisica Nucleare (INFN), Italy; Stichting voor Fundamenteel Onderzoek der Materie (FOM), Nederlandse organisatie voor Wetenschappelijk Onderzoek (NWO), the Netherlands; Council of the President of the Russian Federation for young scientists and leading scientific schools supporting grants, Russia; National Authority for Scientific Research (ANCS - UEFISCDI), Romania; Ministerio de Ciencia e Innovación (MICINN), Prometeo of Generalitat Valenciana and MultiDark, Spain; Agence de l'Oriental and CNRST, Morocco. We also acknowledge the technical support of Ifremer, AIM, and Foselev Marine for the sea operation and the CC-IN2P3 for the computing facilities.

\section{REFERENCES}

Abbasi. R.. Abdou. Y.. Abu-Zayyad. T., et al. 2011. ApJ. 732, 18

Abbasi. R., Ackermann. M., Adams, J., et al. 2009. Phys. Rev. D, 79, 062001

Abdo, A.A, Ackermann, M., Ajello, M., et al. 2011 , ApJ, 734, 28

Adrián-Martínez, S., Ageron, M., Aguilar, J. A., et al. 2012, JINST, 7, T08002

Adrián-Martínez, S., Aguilar. J. A., Al Samarai. I.. et al. 2011, ApJ, 743, L14

Ageron. A.. Aguilar. J. A.. Al Samarai. I.. et al. 2011. Nucl. Instrum. Methods A. 656. 11

Aguilar, J. A., Albert, A., Ameli, F., et al. 2005a, Nucl. Instrum. Methods A. 555,132

Aguilar, J. A., Albert, A., Ameli. F., et al. 2007, Nucl. Instrum. and Methods A. 570,107

Aguilar, J. A., Albert, A., Amram, P., et al. 2005b, Astropart. Phys., 23, 131

Aguilar, J. A., Albert. A., Anton, G., et al. 2010, Astropart. Phys., 34, 179

Aguilar. J. A., Al Samarai, I., Albert, A., et al. 2011a. Astropart. Phys., 34. 539

Aguilar, J. A., A1 Samarai, I., Albert, M., et al. 2011b, Nucl. Instrum. Methods A, 626,128

Aguilar, J. A., \& Hernández-Rey, J. J. 2008, Astropart. Phys., 29, 117

Aharonian. F.. Akhperjanian. A. G., Bazer-Bachi, A. R., et al. 2006. A\&A. 448,43

Aharonian. F.A. Akhperjanian. A. G., Bazer-Bachi, A. R., et al. 2007. A\&A. 464,235

Ambrosio, M., Antolini, R., Auriemma, G., et al. 2001, ApJ, 546, 1038

Amram. P.. Anghinolfi. M.. Anvar. S., et al. 2002. Nucl. Instrum. Methods A. 484, 369

Antonioli, P., Ghetti, C., Korolkova, E. V., Kudryavtsev, V. A., \& Sartorelli, G. 1997, Astropart. Phys.. 7, 357

Bailey, D. 2002. PhD thesis, Univ. Oxford

Barlow. R. 1990. Nucl. Instrum. Methods A. 297. 496

Barr, G. D., Gaisser, T. K., Robbins, S., \& Stanev, T. 2006, Phys. Rev. D, 74. 094009

Becherini, Y., Margiotta, M., Sioli, M., \& Spurio, M. 2006, Astropart. Phys.. 25,1

Bednarek. W.. Burgio, G. F. \& Montaruli. T. 2005. New Astron. Rev.. 49. 1

Berezhko, E. G., \& Volk. H. G. 2008. A\&A. 492, 695

Brunner, J. 2003, in VLVnT Workshop (Amsterdam), ANTARES Simulation Tools, ed. E. de Wolf (Amsterdam: NIKHEF), http://www.vlvnt.nl/ proceedings.pdf

Carminati, G., Margiotta, A.. \& Spurio, M. 2008. Comput. Phys. Commun.. 179,915

Dempster, A. P.. Laird, N. M., \& Rubin, D. B. 1977, J. R. Stat. Soc. B, 39, 1

Feldman, G. J., \& Cousins, R. D. 1998. Phys. Rev. D, 57, 3873

Gould. R. L.. \& Schreder. G. P. 1966. Phys, Rev. Lett., 16. 252

Halzen, F., \& Hooper, D. 2002, Rep. Prog. Phys., 65, 1025

Heijboer, A. J. 2004, PhD thesis, Univ. Amsterdam

Hill, G. C., \& Rawlins, K. 2003, Astropart. Phys., 19, 393

Jelley, J. V. 1966. Phys. Rev. Lett., 16, 479

Kappes, A., Hinton, J., Stegmann, C.. \& Aharonian. F. A. 2007, ApJ, 656, 870

Nikishov, A. I. 1962, Sov. Phys. JETP, 14, 393

Pumplin, J., Stump, D. R., Huston, J., Lai, H. L., et al. 2002, J. High Energy Phys., JHEP07(2002)012

Stecker. F. W. 2005. Phys. Rev. D. 72. 107301

Thrane. E.. Abe, K.. Hayato, Y., et al. 2009. ApJ, 704, 503

Zirakashvili, V. N., \& Aharonian, F. A. 2010, ApJ, 708, 965 